\title{
ADOLESCENTE É TUDO IGUAL? IMPLICAÇÕES SOBRE O COMPORTAMENTO SEXUAL, FATORES ASSOCIADOS E AS PRÁTICAS PREVENTIVAS
}

\section{ARE ADOLESCENTS ALL THE SAME? ISSUES REGARDING SEXUAL BEHAVIOR, SEX-RELATED ASPECTS AND PREVENTION}

\author{
LUIZ ROGÉRIO ROMERO \\ romero@fct.unesp.br \\ Universidade estadual paulista (UNESP) \\ IVETE DALBEN (in memorian) \\ Universidade estadual paulista (UNESP)
}

\begin{abstract}
RESUMO O texto aborda o comportamento sexual de risco na adolescência, enfatizando as relações de gênero, familiar, grupo de amigos, escolhas e possibilidades preventivas. A partir da literatura, aponta-se para a complexidade do comportamento sexual. Do outro lado, enfatizam-se as ações preventivas locais e específicas para a realidade do grupo atendido, considerando a escuta como ponto de partida. A família, grupos de convivência e parcerias intersetoriais podem contribuir na prevenção do sexo de risco na adolescência.
\end{abstract}

PALAVRAS-CHAVE: Comportamento do Adolescente. Educação em Saúde. Promoção da Saúde.

ABSTRACT The paper deals with risky sexual behavior during adolescence, pointing out gender and family relationships, friendship, choices and prevention. The complexity of sexual behavior is approached based on literature. On the other hand, local and specific preventive actions taking into account the reality of the group at issue are pointed out, taking listening as the starting point. Family, social interaction and multidisciplinary associations can all contribute to preventing risky sexual behavior during adolescence.

KEYWORDS: Adolescent behavior. Health Education. Health Promotion.

\section{INTRODUÇÃO}

A segunda década de vida apresenta aos indivíduos cenários de descobertas e mudanças biopsicossociais, além de outros determinantes para os anos a seguir. A interpretação desta fase tem sido construída ao longo da história da civilização, assim como a autopercepção do jovem, relações com a família, amigos e sociedade. 
Neste período de exercício da autonomia, algumas situações específicas de risco à saúde são intensificadas e muitas vezes simultâneas, como o uso de substâncias psicoativas e a iniciação sexual desprotegida.

Soma-se ainda o fato de que ações preventivas generalizadas e destinadas aos jovens têm demonstrado constantes limitações e baixa efetividade. Contudo, diversos trabalhos científicos têm enfocado a questão, apresentando dados sobre as práticas sexuais de risco, críticas aos modelos de prevenção, assim como subsídios para a qualificação das estratégias de educação em saúde do adolescente.

Desse modo, o presente texto pretende contribuir com a reflexão sobre a problemática do comportamento sexual de risco, enfatizando as relações de gênero, familiar, grupo de amigos, escolhas e possibilidades de prevenção.

\section{COMPORTAMENTO SEXUAL E AS RELAÇÕES DE GÊNERO NA ADOLESCÊNCIA}

Pretende-se neste tópico, discutir aspectos referentes ao conhecimento sobre prevenção, comunicação e negociação do uso de preservativos. Embora esta ação seja amplamente disseminada pelas campanhas de prevenção, sobretudo no período de carnaval, a decisão do uso parece sofrer interferência de outras variáveis além do conhecimento.

Borges (2007) contribui, a este respeito, em estudo sobre relações de gênero e iniciação sexual de mulheres adolescentes. Dentre os resultados, relata diminuição de uso de preservativos nas práticas sexuais que envolvem o aspecto afetivo e amoroso. Este panorama configura-se nas diferenças observadas em relação ao comportamento sexual masculino e feminino. Vilela e Doreto (2006) assinalam que nas relações de gênero espera-se que as meninas sejam recatas e no âmbito sexual e valorizem a virgindade. Em contrapartida, para os meninos, compreende-se a facilidade na abordagem do tema e suas vivências sexuais. Nesta perspectiva, o diálogo sobre métodos preventivos na relação sexual torna-se dificultado, assim como as trocas de informações sobre iniciação sexual segura.

Aponta-se também a relação de submissão, cabendo à mulher conceder o ato sexual. Geluda et al. (2006) ressaltam que para as mulheres, a exposição ao risco 
parece ser a melhor opção comparando a possibilidade de perder a relação estável ou inicial.

Borges e Schor (2005) acrescentam que os parceiros na iniciação sexual feminina sejam, aproximadamente, quatro anos mais velhos. Este fato poderia dificultar a solicitação de uso de preservativo considerando as desigualdades de relação de gêneros e vulnerabilidade referida. Salientam ainda que metade dos jovens investigados iniciaram a vida sexual sem o uso de métodos preventivos. Corroboram estudos anteriores ao afirmar que existem outras variáveis envolvidas na decisão de usar ou não usar preservativos além do conhecimento sobre métodos preventivos. Aspectos importantes do comportamento sexual diferem entre gêneros, sendo indicativos de necessidade de intervenções específicas quanto à saúde reprodutiva.

Nas relações de gênero, a confiança e a fidelidade são pressupostos estabelecidos para a relação sexual sem o uso de preservativo. Vilela e Doreto (2006) apontam que a fidelidade ao parceiro oferece a segurança necessária para a relação sexual sem o uso de preservativo. Compreende-se que no universo feminino, uma relação estável e monogâmica é necessária para o bom desenvolvimento de seu papel social e a manutenção deste comportamento contribui para o desenvolvimento e ascensão de sua autoestima. Segundo os autores, percebe-se nos discursos dos adolescentes, a fidelidade feminina como obrigação, ao passo que a infidelidade masculina seria esperada. A confiança recíproca entre os parceiros e respectiva fidelidade parece dificultar a solicitação do uso de preservativo. Para estes adolescentes, o pedido seria o mesmo que duvidar do outro ou assumir a quebra da lealdade. De fato, este entendimento social disseminado entre os jovens tem sustentado o aumento da incidência de contaminação pelo HIV, sobretudo entre as mulheres em relações estáveis. O uso de preservativo muitas vezes relaciona-se ao sexo de risco, desconfiança e prostituição. Este ponto de vista foi reforçado anteriormente pelas estratégias de prevenção focadas nas relações sexuais com múltiplos parceiros, infidelidade, e "garotas de programa", considerando que as mulheres em relações estáveis estivessem livres do risco de contaminação pela Aids. 
Outros estudos reforçam esta assertiva. Sousa, Espírito Santo e Motta (2008) ao estudarem práticas de prevenção do HIVIAids em mulheres moradoras de um bairro de Teresina, Piauí, evidenciam que a grande maioria não utilizava o preservativo durante as relações sexuais. A confiança na relação estável com o parceiro e a autoavaliação de baixa vulnerabilidade de infecção por DST/Aids foram as principais justificativas. Entre aquelas que utilizam, prevaleceu o uso intermitente em relação ao comportamento preventivo em todas as relações sexuais.

Stulhofer et al. (2009) apontam que mais de $20 \%$ dos jovens participantes do estudo não usaram nenhum método contraceptivo na primeira relação sexual. Enfatizam também a necessidade de que programas de prevenção abordem o uso de preservativos, principalmente com mulheres, considerando a baixa frequência.

Segundo Ministério da Saúde (BRASIL, 2008a), em estudo sobre prevalência e frequências relativas de DST em populações selecionadas de seis capitais brasileiras (Fortaleza/CE; Goiânia/GO; Manaus/AM; Porto Alegre/RS; Rio de Janeiro/RJ; São Paulo/SP), cerca de 79\% das gestantes relataram atividade sexual com um único parceiro nos últimos 12 meses, 15,8\% dois a cinco parceiros e 0,8\% mais de cinco neste período. Dentre esta população, 6,2\% referiram usar preservativo com parceiro fixo, $43,5 \%$ usa às vezes e $49,2 \%$ relataram nunca usar preservativos.

Estes dados enfocam a diversidade de situações e comportamentos determinantes no processo preventivo. Berten e Rossem (2009) apontam a necessidade de estudar este cenário considerando características de subgrupos, seja relativa ao sexo, idade, aspectos demográficos e/ou religiosos, pois a variação de conhecimento e comportamento pode ser muito diferente para o mesmo grupo de adolescentes.

Segundo a literatura apresentada, o risco de exposição às práticas sexuais não seguras, além do conhecimento, está associado à afetividade nas relações, aos diferentes papéis sociais atribuídos entre homens e mulheres, limitações no diálogo, assim como a perda da estabilidade no relacionamento. O uso do preservativo ou solicitação desta conduta seria assumir a desconfiança. Este fato relaciona-se com a maior prevalência de uso intermitente do preservativo entre os adolescentes em parcerias estáveis. Portanto, as ações preventivas devem se aproximar da 
complexidade e dos determinantes do tema, suplementando o enfoque informativo unidirecional e de culpabilização do indivíduo. Neste caminho, a família e o grupo de amigos exercem importante influência e devem ser considerados no panorama da prevenção.

\section{O AMBIENTE FAMILIAR, AMIGOS E AS ESCOLHAS SOB INFLUÊNCIAS AFETIVAS}

Em relação ao envolvimento paternal e sua aproximação do cotidiano dos filhos adolescentes, Vukovic e Bjegovic (2007) ao estudar adolescentes sérvios, afirmam que dificuldades de diálogo com os pais ou morar com apenas um deles associa-se à prática sexual sem o uso de métodos contraceptivos.

Outro estudo utilizou dados de amostra representativa composta por 8.984 norte-americanos entre 12 e 16 anos de idade. Investigaram anualmente a influência dos pais no comportamento sexual de risco durante a adolescência. Os resultados revelam que durante este período, tanto pais quanto adolescentes apresentam mudanças. Os filhos procuram atividades envolvendo grandes emoções e exercitam a autonomia, dedicam mais tempo aos grupos de amigos e iniciam os primeiros envolvimentos românticos e sexuais. Já os pais se reorganizam para estas mudanças exercendo acompanhamento desta autonomia de forma mais distante. Destacam ainda que em alguns casos, a ocorrência de dificuldades e/ou rompimento na relação entre pais e filhos. Por outro lado, o envolvimento paternal e sua aproximação do cotidiano dos filhos adolescentes contribuem positivamente como proteção aos comportamentos sexuais de risco (COLEY; VOTRUBA-DRZAL; SCHINDLER, 2009).

A discussão e conversa com pais tem-se mostrado como fator positivo para aumento de conhecimento sobre HIVIAids, embora, nesta fase da vida, a influência e opiniões do grupo de amigos tenham maiores forças relativas ao comportamento sexual. Dessa forma, atualmente tem se sugerido ampliação de espaço para discussões abertas sobre este tema, de forma a favorecer a eliminação de dúvidas e subsidiar conhecimentos adequados sobre comportamento sexual e respectivas atitudes preventivas (BERTEN; ROSSEM, 2009). 
Baseado nas estatísticas de morbidade e mortalidade entre jovens norteamericanos, Casey, Jones e Hare (2008) salientam a importância de se compreender as escolhas dos adolescentes por comportamentos de risco à saúde. Os autores defendem que o desenvolvimento neurológico não ocorre de modo linear, caracterizando a adolescência como momento de maior aproximação e exposição de situações de risco à saúde, se comparado à infância e a vida adulta. Apoiado em estudos anteriores, demonstra-se que os adolescentes se expõem ao risco conscientemente, sob influência emocional, afetiva, sentimentos e do grupo de amigos. Em outro trabalho, Bell (2009) descreve a influência de sentimentos como vergonha ou timidez em relação à aquisição e uso de preservativo, em estudo realizado entre jovens de 12 a 17 anos de áreas rurais e litorâneas da Inglaterra. Este sentimento associa-se à dificuldade de relacionamento e de autoconfiança. Segundo o autor, esta característica se aplica aos jovens e adultos de ambos os sexos. Considera evidente a necessidade de acesso aos preservativos sem que o adolescente se sinta em exposição pessoal. Outro fato importante são campanhas preventivas e programas de orientação sexual preconizar escolhas e decisões racionais para situações de relacionamento que envolvem o âmbito emocional.

Somam-se, neste contexto, os achados de Giannotta et al. (2009). Avaliaram no estudo o significado da relação sexual entre 208 escolares italianos de 14 a 19 anos de idade e de ambos os sexos. Com base em aplicação de questionários, sugere-se que a relação sexual para o adolescente está ligada a muitas outras variáveis. Entre os tópicos apontados, além do amor, reprodução das práticas dos grupos de amigos, satisfação de desejos pessoais ou para contradizer a normalidade esperada, com significado de transgressão. Segundo os autores, adolescentes que referem significado social negativo para a prática sexual são associados à virgindade ou menor número de parceiros sexuais. No entanto, foram mais suscetíveis a não usar métodos contraceptivos e ter praticado sexo com parceiros ocasionais nos últimos encontros.

Esta prática relaciona-se à provável pressão do grupo para a iniciação sexual, e neste encaminhamento, a eventual desconsideração de métodos preventivos e dos riscos à saúde agindo por impulsos emocionais. Desse modo, a pressão do 
grupo pode ser fator de risco para práticas de sexo não seguro, assim como para o uso de álcool, tabaco e maconha entre adolescentes.

\section{O INÍCIO DA VIDA SEXUAL E OS FATORES RELACIONADOS}

A crescente precocidade da idade de iniciação sexual tem sido observada em muitos países. Borges e Schor (2005) estudaram as relações de gênero na iniciação sexual de crianças e adolescentes da cidade de São Paulo. Apontaram tendência de antecipação da idade nesta prática, principalmente em relação ao sexo feminino, aproximando-se da faixa etária apresentada pelos homens. Barbosa e Koyama (2008), ao estudar o comportamento e práticas sexuais de homens e mulheres no Brasil entre 1998 e 2005, revelam a existência de padrão de mudança caracterizado por aumento da proporção de mulheres que iniciam a vida sexual entre 16 e 19 anos.

Em outro contexto, Laflin, Wang e Barry (2008) investigaram características demográficas e psicossociais preditoras da iniciação sexual precoce. Em pesquisa longitudinal entre 884 estudantes de escolas suburbanas e rurais do oeste dos Estados Unidos, reforçam que os homens têm reportado iniciação sexual precoce em proporção superior às mulheres. Apontam ainda que bom desempenho acadêmico, ter expectativas de futuro, religiosidade e estrutura familiar tradicional estão associados ao adiamento de atividades sexual em adolescentes.

Outro estudo longitudinal envolvendo 694 crianças, pré-adolescentes e adolescentes norte-americanos de ambos os sexos, relacionou o início da vida sexual aos comportamentos sociais desta população. Foi encontrada correlação entre agressividade na infância, dificuldades no ambiente escolar, comportamentos antissociais e início precoce de atividades sexuais. Destaca, ainda, o uso de substâncias psicoativas na infância como forte preditor de atividade sexual precoce (SCHOFIELD et al., 2008).

Price e Hyde (2009) investigaram a acumulação de fatores de risco para a iniciação sexual relacionados ao indivíduo, família e outros fatores socioculturais. $O$ estudo utilizou-se de entrevistas entre 273 adolescentes norte-americanos de ambos os gêneros e média de idade de 15,5 anos. Os resultados apontam que a iniciação 
precoce de atividade sexual para mulheres associa-se, entre outras variáveis, a extensos períodos assistindo televisão, baixa autoestima, relacionamento limitado com os pais, baixo rendimento acadêmico e baixa escolaridade dos pais. Para os homens, a iniciação sexual precoce foi relacionada ao avançado desenvolvimento maturacional, tempo assistindo televisão e relacionamento limitado com os pais. A participação em atividades esportivas e depressão não se relacionaram à iniciação sexual precoce. Os autores sugerem que os programas de intervenção considerem a complexidade relacionada ao comportamento sexual e aborde nas ações preventivas, outros fatores influentes no comportamento de saúde de adolescentes. Também indicam a possibilidade de incluir a participação dos pais nos programas de prevenção, assim como o encorajamento destes em promover apoio e orientações aos adolescentes.

Manlove et al. (2009) analisaram dados de 1992, 1997 e 2002 sobre crescimento familiar e tendências de comportamentos de saúde reprodutiva de jovens norte-americanos. Sugerem que o ambiente familiar e aumento da idade para o início da prática sexual pode auxiliar na melhora do padrão de saúde reprodutiva entre adolescentes. O estudo reforça a necessidade de organização de programas de orientação que considerem características familiares, individuais e ambientes de convivência para subsidiar decisões sobre sexo e contracepção na juventude.

Cavazos-Rehg et al. (2009) estudaram a relação entre idade do inicio da prática sexual, gênero e etnia, entre adolescentes norte-americanos no período de 1999-2007. Dentre os destaques, aponta-se para a diferença significativa entre gênero, etnia e o início da vida sexual. Outros fatores ainda pouco compreendidos também se relacionam como as expectativas sociais e o contexto cultural. Os autores indicam a necessidade de atentar para essas diferenças na elaboração de programas de educação sexual adequando-se às características do grupo em questão.

\section{A PRÁTICA SEXUAL E A PREVENÇÃO NA ADOLESCÊNCIA}

No Brasil, Doreto e Vieira (2007), ao estudarem noventa adolescentes do sexo feminino de baixa renda, no município de Ribeirão Preto, São Paulo, estimaram 
que $93,3 \%$ das entrevistadas iniciaram a vida sexual. O uso do preservativo foi apontado como a principal forma de evitar as DST, no entanto, apenas $35,2 \%$ relataram usar sempre, $38,9 \%$ às vezes e $25,9 \%$ disseram nunca usar. Salientam ainda que $37,1 \%$ não fizeram uso de preservativo em sua última relação sexual, sendo que $29,4 \%$ alegaram confiança no parceiro e $26,5 \%$ uso de anticoncepcional oral. Dessa forma, entende-se que adolescência configura-se momento de risco e com necessidade de atenção diferenciada, pois iniciaram a vida sexual precocemente, apresentaram baixo conhecimento sobre DST/Aids e ampla vulnerabilidade devido à ausência de práticas constantes e efetivas de prevenção.

Camargo e Ferrari (2009) analisaram os conhecimentos de adolescentes sobre sexualidade, métodos contraceptivos, gravidez, DST, e Aids, antes e após oficinas de prevenção. O trabalho foi realizado com 117 estudantes da oitava série de uma escola estadual em Londrina, Paraná. Apontaram para a diminuição da idade média de inicio de práticas sexuais. Destacaram que a diminuição da idade média para entrada na puberdade está antecedendo ao desenvolvimento cognitivo e emocional esperado para experiências sexuais. Estes dados foram entendidos como fatores de risco para gravidez não planejada e DST, considerando que o uso de métodos preventivos consistentes não tem sido relatado por esta população. Apontaram também para as relações de gênero, sendo as mulheres mais vulneráveis na discussão sobre o uso de camisinha e os homens na busca de reforçar sua masculinidade através do sexo. Embora se tenha avançado em relação aos conhecimentos por meio dessas oficinas, ainda assim os adolescentes mantiveram dúvidas sobre ciclo menstrual e formas de contágio e prevenção às DST. Os autores reportaram a necessidade de proporcionar espaços de discussão e diálogo, a partir da realidade dos adolescentes, assim como envolvimento de parceiros e familiares nesta dinâmica preventiva.

Em pesquisa sobre comportamento de risco entre jovens norte-americanos, ressaltou-se que $46,8 \%$ dos adolescentes em idade escolar já tiveram relações sexuais. Destes, 3,7\% das mulheres e 8,8\% dos homens iniciaram antes dos 13 anos de idade. Cerca de $12 \%$ da população feminina e $16,5 \%$ da masculina tiveram quatro ou mais parceiros em relações sexuais durante a vida. $\mathrm{O}$ uso de preservativo foi apontado por $55,9 \%$ das mulheres e $70 \%$ dos homens. Salientou-se que $19 \%$ das 
jovens e 27,6\% dos homens relataram o uso de álcool ou drogas antes da última relação sexual. O estudo ainda investigou programas de prevenção de DST e HIV/Aids nas escolas. Dentre os estudantes, $87,9 \%$ relataram ter participado deste tipo de orientação (EATON et al., 2006).

Investigando a trajetória do comportamento sexual de indivíduos holandeses entre 12 e 25 anos de idade, foram definidos dois padrões de experiências, sendo o primeiro caracterizado por sequência progressiva de intimidade (beijar; acariciar; relação sexual). Outras sequências foram descritas como irregulares. Observou-se que indivíduos com trajetória progressiva tendem a apresentar comportamento sexual mais seguro. Dentre as atitudes, notou-se uso de métodos contraceptivos, principalmente preservativos, com maior frequência. Já os que apresentaram trajetória irregular eram mais suscetíveis à prática sexual sem o uso de preservativo. Destacou-se que a população feminina deste grupo apresentou maior risco de prática de sexo sem o uso de preservativo. Os autores indicaram que os futuros programas de prevenção devem levar em consideração estas sequência de comportamento, sobretudo abordar e orientar adolescentes em maior exposição. Destacaram ainda, que os pais, os professores e os serviços de saúde deveriam subsidiar estes jovens com informações sobre a prática sexual segura (GRAF et al., 2009).

Miranda, Gadelha e Szwarcwald (2005) contribuem ao ressaltar a maior vulnerabilidade às doenças sexualmente transmissíveis neste grupo populacional. Em relação à prática sexual de risco, Vilela e Doreto (2006) destacaram a vulnerabilidade como produto da interação entre características do indivíduo, como cognição, afeto e psiquismo e estruturas sociais de desigualdade, entre elas, gênero, classe e raça. Estas variáveis determinariam acessos e oportunidades de informação.

Em outro estudo entre 1.594 adolescentes de 12 a 19 anos de escolas públicas e privadas do município de São Paulo sobre fatores associados aos conhecimentos e ao uso consistente do preservativo masculino, apontou-se que a maior escolaridade, ser do sexo feminino, estudar em escola privada, cor branca e solteiro foram fatores preditores positivos de maior conhecimento sobre DST/Aids (MARTINS et al., 2006). 
Camargo e Botelho (2007) investigaram as experiências sexuais e as atitudes de risco para transmissão do HIVIAids entre 1.386 estudantes da rede pública e particular de três cidades do estado de Santa Catarina. Observou-se que a conduta de risco de prática sexual sem o uso de preservativo foi de $58,5 \%$ para os adolescentes que tiveram relação sexual nos últimos 12 meses. Salientaram ainda que quanto mais experiências sexuais, maior a proporção sexo sem preservativo.

Em 2006, foram publicadas as Diretrizes para Implantação do Projeto Saúde e Prevenção nas escolas (BRASIL, 2006a). Ressaltaram que a faixa etária de 10 a 24 anos corresponde a mais de $30 \%$ da população. Reconheceram também que estes indivíduos estão expostos a diversos fatores de risco à saúde. A vulnerabilidade foi conceituada como chance de exposição ao adoecimento, assim como resultante de conjunto de aspectos individuais, coletivos e contextuais. Acrescentaram ainda que a maior suscetibilidade ao adoecimento relacionou-se a menor disponibilidade de recursos de proteção.

Evans et al. (2004), em estudo sobre riscos específicos entre adolescentes sexualmente ativos, encontraram maior vulnerabilidade relacionada à população negra, gênero masculino, idade de 15 a 18 anos e baixo nível socioeconômico. Notaram também a relação dos conhecimentos e valores do grupo de amigos e sua percepção de comportamento de risco. A participação dos pais na informação sobre comportamento sexual se apresentou como fator protetor ao engajamento em comportamento de risco sexual.

Ayres (2002) muito contribui com a discussão ao destacar entre os aspectos de vulnerabilidade: a pobreza, a exclusão de base racial, a rigidez de papéis nas relações de gênero, a intolerância à diversidade, o limitado diálogo com as novas gerações e a incompreensão dos seus valores e projetos, assim como a desassistência com as gerações mais idosas e a desintegração da sociedade civil no mundo globalizado. Apontou algumas lições aprendidas no campo da prevenção, destacando que "o terrorismo não funciona". Esta frase indicou reflexão sobre as primeiras estratégias e prevenção, baseadas no susto e no medo, e que não tiveram efeitos positivos. Inversamente, este cenário afastou a população das discussões efetivas e subsequentes mudanças necessárias de comportamento. Em relação aos caminhos a percorrer na prevenção, o autor sinalizou para a necessidade de 
aproximação e criação de espaços de diálogos e compreensão de subjetividades em substituição de ações puramente informativas. Salientou que grau de escolarização, origem étnica e cultura religiosa, devem sempre ser considerados no entendimento do cenário e nas estratégias preventivas.

Em revisão de literatura sobre programas de redução de comportamento de risco à DST/AIDS e gravidez na adolescência, foram destacados quatro fatores diretamente relacionados à efetividade da intervenção: i) o foco no risco específico a ser abordado; ii) duração e intensidade do programa; iii) determinação cuidadosa da constituição do programa na totalidade e; iv) treinamento de facilitadores de grupo (ROBIN et al., 2004).

Vilela e Doreto (2006) contribuem ainda sobre programas efetivos de prevenção. Apontam que as informações se difundem de modo distinto pelas camadas sociais e são elaboradas contextualmente. Isso permite que o pensamento do adequado ou não na adolescência seja vivenciado de maneira particular nos diversos grupos. Desse modo, para analisar a atividade sexual dos jovens, seria importante considerar como cada um se situa em relação a si mesmo e a sua capacidade de responder pelos seus atos, tendo por referência os diferentes sentidos sociais atribuídos à adolescência.

\section{CONSIDERAÇÕES}

Observando os apontamentos dos textos analisados, tem-se a ideia da complexidade referente ao assunto "prática sexual na adolescência". O comportamento preventivo muitas vezes situa-se na relação entre o conhecimento sobre prevenção, aspectos emocionais, afetivos, relações de gênero, confiança e fidelidade, compreensão e expectativas em torno do ato sexual, aceitação no grupo de amigos e a questão: usar ou não usar camisinha?

Neste dilema multifacetado, o uso de substâncias psicoativas e a precocidade da iniciação sexual atenuam a racionalização e percepção dos riscos, dificultam o estabelecimento de condutas preventivas e potencializam ainda mais as práticas sexuais não seguras. Entende-se, desta maneira, que a ação preventiva deve ir além de informar. 
O fato de ensinar como se usa o preservativo e enfatizar a proteção oferecida, embora seja muito importante, não ameniza diretamente os riscos à saúde sexual e reprodutiva. Também a prevenção unidirecional, massiva, padronizada e imersa em estereótipos não tem se mostrado eficaz ao longo do tempo.

Portanto, sugere-se que a prevenção tenha como foco o contexto local. Ressalta-se como preponderante $\mathrm{o}$ respeito às características intrínsecas e específicas da realidade do grupo que será atendido. Deve-se reconhecer as necessidades e indagações dos participantes, valorizando a escuta como relevante fonte de informações.

Também os aspectos familiares, ambientais e grupos de convivências demonstraram relevância na compreensão de comportamentos de saúde. 0 reconhecimento das reais necessidades individuais e coletivas e adequada aproximação desses jovens parecem situar-se como diretrizes no trabalho em saúde do adolescente. O envolvimento da família e dos amigos poderia auxiliar na identificação de caminhos potenciais de ações preventivas.

Nesta tarefa, a organização de ações intersetoriais torna-se premissa para a qualificação, abrangência e especificidades necessárias para o trabalho preventivo. Setores ligados aos serviços públicos de saúde, educação, esporte, assistência social, além de associação de moradores, organizações não governamentais, entre outros, poderiam apresentar suas possibilidades de trabalho conjunto e integrado na promoção da saúde do adolescente.

\section{LUIZ ROGÉRIO ROMERO}

Doutor em Saúde Coletiva. Professor da Universidade Estadual Paulista (UNESP). Departamento de Educação Física, Faculdade de Ciências e Tecnologia de Presidente Prudente, São Paulo.

\section{IVETE DALBEN}

Médica. Pós-doutora em Epidemiologia. Professora da Universidade Estadual Paulista (UNESP).

\section{REFERÊNCIAS}

AYRES, J. R. C. M. Práticas educativas e prevenção de HIV/Aids: lições aprendidas e desafios atuais. Comunic, Saúde, Educ, v. 6, n. 11, p.11-24, ago. 2002. 
BARBOSA, R. M.; KOYAMA, M. A. H. Comportamento e práticas sexuais de homens e mulheres, Brasil 1998 e 2005. Rev. Saúde Pública, v. 42, Supl 1, p. 21-33, 2008.

BELL, J. Why embarrassment inhibits the acquisition and use of condoms: A qualitative approach to understanding risky sexual behaviour. J. Adolesc. v. 32, n.2, p. 379-391, 2009.

BERTEN, H.; ROSSEM, R. V. Doing worse but knowing better: An exploration of the relationship between HIVIAids knowledge and sexual behavior among adolescents in Flemish secondary schools. J. Adolesc., v. 32, n.5, p. 1303-1319, 2009.

BORGES, A. L. V. Relações de gênero e iniciação sexual de mulheres adolescentes. Rev. Esc. Enferm. USP, v. 41, n. 4, p. 597-604, 2007.

BORGES, A. L. V.; SCHOR, N. Trajetórias afetivo-amorosas e perfil reprodutivo de mulheres adolescentes residentes no Município de São Paulo. Rev. Bras. Saúde Mater. Infant., Recife, v. 5, n. 2, p. 163-170, jun. 2005.

BRASIL. Ministério da Saúde. Secretaria de Vigilância em Saúde. Programa Nacional de DST e Aids. Diretrizes para implantação do Projeto Saúde e Prevenção nas Escolas. Brasília, 2006. 24p. (Série A. Normas e Manuais Técnicos)

Ministério da Saúde. Secretaria de Vigilância em Saúde. Programa Nacional de DST e Aids. Prevalências e freqüências relativas de Doenças Sexualmente Transmissíveis (DST) em populações selecionadas de seis capitais brasileiras, 2005. Brasília, 2008. 224 p. (Série G. Estatística e Informação em Saúde).

CAMARGO, B. V.; BOTELHO, L. J. Aids, sexualidade e atitudes de adolescentes sobre proteção contra o HIV. Rev. Saúde Pública, v. 41, n.1, p. 61-68, fev. 2007.

CAMARGO, E. A. I.; FERRARI, R. A. P. Adolescentes: conhecimentos sobre sexualidade antes e após a participação em oficinas de prevenção. Ciênc. Saúde Colet., v. 14, n. 3, p. 937-946, 2009.

CASEY, B. J.; JONES, R. M.; HARE, T. A. The Adolescent Brain. Ann. N.Y. Acad. Sci., v. 11, n.24, p. 111-126, 2008.

CAVAZOS-REHG, P. A. et al. Age of sexual debut among US adolescents. Contraception, v. 80, n.2, p. 158-162, 2009.

COLEY, R. L.; VOTRUBA-DRZAL, E.; SCHINDLER, H. S. Fathers' and Mothers' Parenting Predicting and Responding to Adolescent Sexual Risk Behaviors. Child. Development, v. 80, n. 3, p. 808-827, mai./jun. 2009.

DORETO, D. T.; VIEIRA, E. M. O conhecimento sobre doenças sexualmente transmissíveis entre adolescentes de baixa renda em Ribeirão Preto, São Paulo, Brasil. Cad. Saúde Pública, Rio de Janeiro, v. 23, n. 10, out. 2007. 
EATON, D. K. et al. Youth Risk Behavior Surveillance - United States, 2005. MMWR Surveill. Summ., v. 55, n. 5, p. 1-108, jun. 2006.

EVANS, A. E. et al. An Exploration of the Relationship Between Youth Assets and Engagement in Risky Sexual Behaviors. J. Adolesc. Health, v. 35, n.5, p. 424-430, 2004.

GELUDA, K. et al. "Quando um não quer, dois não brigam": um estudo sobre o não uso constante de preservativo masculino por adolescentes do Município do Rio de Janeiro, Brasil. Cad. Saúde Pública, Rio de Janeiro, v. 22, n. 8, p. 1671-1680, ago. 2006.

GIANNOTTA, F. et al. Meanings of sexual intercourse for Italian adolescents. J. Adolesc., v. 32, p. 157-169, 2009.

GRAAF, H. et al. Sexual Trajectories during Adolescence: Relation to Demographic Characteristics and Sexual Risk. Arch. Sex. Behav., v. 38, n.2, p. 276-282, 2009.

LAFLIN, M. T.; WANG, J.; BARRY, M. A Longitudinal Study of Adolescent Transition from Virgin to Nonvirgin Status. J. Adolesc. Health, v. 42, n.3, p. 228-236, 2008.

MANLOVE, J. et al. Trends in Sexual Experience, Contraceptive Use, and Teenage Childbearing: 1992-2002. J. Adolesc. Health, v. 44, n.5, p. 413-423, 2009.

MARTINS, L. B. M. et al. Fatores associados ao uso de preservativo masculino e ao conhecimento sobre DST/Aids em adolescentes de escolas públicas e privadas do Município de São Paulo, Brasil. Cad. Saúde Pública, v. 22, n. 2, p. 315-323, 2006.

MIRANDA, A. E.; GADELHA, A. M. J.; SZWARCWALD, C. L. Padrão de comportamento relacionado às práticas sexuais e ao uso de drogas de adolescentes do sexo feminino residentes em Vitória, Espírito Santo, Brasil, 2002. Cad. Saúde Pública, v. 21, n. 1, p. 207-216, 2005.

PRICE, M. N.; HYDE, J. S. When Two Isn't Better Than One: Predictors of Early Sexual Activity in Adolescence Using a Cumulative Risk Model. J. Youth Adolesc., v. 38, n.8, p. 1059-1071, 2009.

ROBIN, L., et al. Behavioral Interventions to Reduce Incidence of HIV, STD, and Pregnancy Among Adolescents: A Decade in Review. J. Adolesc. Health. v. 34, n.1, p. 3-26, 2004.

SCHOFIELD, H. L. T. et al. Predicting Early Sexual Activity with Behavior Problems Exhibited at School Entry and in Early Adolescence. J. Abnorm. Child. Psychol., v. 36, n.8, p. 1175-1188, 2008. 
SOUSA, M. C. P.; ESPIRITO SANTO, A. C. G.; MOTTA, S. K. A. Gênero, vulnerabilidade das mulheres ao HIV/Aids e ações de prevenção em bairro da periferia de Teresina, Piauí, Brasil. Saúde soc., São Paulo, v. 17, n. 2, p.58-68, jun. 2008.

STULHOFER, A. et al. An Assessment of HIVISTI Vulnerability and Related Sexual Risk-Taking in a Nationally Representative Sample of Young Croatian Adults. Arch. Sex. Behav., v. 38, n.2, p. 209-225, 2009.

VILELLA, W. V.; DORETO, D. T. Sobre a experiência sexual dos jovens. Cad. Saúde Pública., v. 22, n. 11, p. 2467-2472, 2006.

VUKOVIC, D. S.; BJEGOVIC, V. M. Risky sexual behavior of adolescents in Belgrade: Association with Socioeconomic status and family structure. J. Adolesc., v. 30, p. 869-877, out. 2007. 